\title{
Modelling Operational Decision-Making in Agriculture
}

\section{Roger Martin-Clouaire}

Mathématiques et Informatique Appliquées de Toulouse, Université de Toulouse, Institut National de la Recherche Agronomique, Castanet Tolosan, France

Email: roger.martin-clouaire@inra.fr

How to cite this paper: Martin-Clouaire, R. (2017) Modelling Operational DecisionMaking in Agriculture. Agricultural Sciences, 8, 527-544.

https://doi.org/10.4236/as.2017.87040

Received: May 31, 2017

Accepted: July 1, 2017

Published: July 4, 2017

Copyright (C) 2017 by author and Scientific Research Publishing Inc. This work is licensed under the Creative Commons Attribution International License (CC BY 4.0).

http://creativecommons.org/licenses/by/4.0/

\begin{abstract}
Farm management practices differ considerably among farmers. In this article, we explore the processes which farmers go through when making operational decisions about technical interventions. Because farmers have different approaches to the decision-making process, it is essential to describe these differences to identify areas in which management skills require improvement. This study identifies and represents contextual, informational and inferential aspects of the cognitive work a farm manager performs in operational decision-making. We developed a conceptual modelling framework that structures the decision-making behaviour along a set of cognitive processes such as perception, interpretation, goal reasoning, planning and judgment. These processes are activated repeatedly throughout the production process. The framework can help characterize variation in management behaviour and performance, and identify promising directions for improvement.
\end{abstract}

\section{Keywords}

Belief, Goal, Intention, Plan, Event, Agricultural Production Management

\section{Introduction}

Most daily tasks of a crop or animal farm are initiated by the farm manager. Decisions directly connected to actions (hereafter called "operational decisions") strongly influence the type, quality and quantity of agricultural output and can have major economic and environmental consequences. Operational decisionmaking has always been an integral and engaging part of a farmer's job. Its importance has increased due to technological changes in the agricultural sector, commercial competition, and the more stringent sociological and environmental requirements that farmers must integrate as managers. Agricultural production is a decision-intensive undertaking. Examples of decisions that occupy most 
farmers' daily routines include which crops to plant, which inputs to use, when to plow, when to sow, when to irrigate, how much crop to sell and how much crop to store for later sale. Agriculture is truly unique in that farmers and households make these decisions themselves or in consultation with relatively small numbers of neighbors, friends, or partners. The primary drivers of decisions are the farmer's motives, beliefs, perceptions and preferences. Consequently, farmers' decisions are heterogeneous from farm to farm and even from field to field: not all farmers in a region grow the same crops or manage their livestock in the same way, and decisions depend on situations that change continually and are never exactly the same twice.

Due to disciplinary bias, most studies of decision-making characteristics tend to adopt particular perspectives and factors (e.g., biophysical, economic, psychological, cultural, social) at the expense of others. Many researchers and policy-makers tend to view farm production through a simplistic economic lens which assumes that all farmers have similar personal and business goals and focus on managing their farms essentially as profit-driven businesses [1]. These studies focus on the performance of statistical composites rather than on individual performance. Other studies analyse the various factors that influence farmers' decisions [2] [3] [4] [5]. These include the farmers' education, the path of their professional experience, their attitudes towards the environment, their personal desires and preferences, and farm characteristics (e.g. size, land characteristics, equipment). Socio-economic factors are also important, and include professional relationships (e.g. information networks, advisory services, and market-based networks), household characteristics (e.g. degree of pluriactivity, involvement of other family members) and the financial situation. These studies provide useful insights into the roles of these factors in adapting their production systems (e.g. strategies to cope with climate change) but do not consider the dynamic processes by which actions are decided in specific situations. Schlüter et al. [6] proposed a theoretical framework that can accommodate various decision-making theories to address natural resource problems and study humanenvironment interactions within this field. This framework considers single time step decision problems and is therefore not appropriate for sequential decision problems such as those involved in the management of agricultural production processes.

Better understanding of decision-making mechanisms would help explain certain inadequate performances and predict how agricultural management practices could change when external circumstances change. This understanding is a prerequisite for designing strategies or policies to improve farmers' production-management skills. By relying on overly narrow definitions of rationality, previous studies of farmers' decisions often failed to identify the complexity of farmers' operational decision-making. The most overlooked aspects include the difficulty in obtaining up-to-date information about the biophysical system; the multiple, nondeterministic and difficult-to-reverse effects of most agricultural operations; and the time lag between their execution and the resulting conse- 
quences. Little attention is given to the agronomic and organisational need to spatially and temporally coordinate cropping operations. Because changes in the biophysical state and the external environment are beyond the farmer's control, they have considerable implications for the farmer's response to the changing reality. The decision-making process must be considered in terms of its ability to invoke behaviour that is reactive to contingencies and is proactive, i.e., influenced by goals and plans.

Farmers' management behaviour is difficult to understand by simply observing their actions, even over a long period. Understanding their productionmanagement practices more thoroughly requires understanding how they think, what they know, what matters to them, what attracts their attention, how they organise information and the feelings that underlie their decision-making processes. This article is a theoretical description of primitive modelling notions used to characterize and analyse farmers' management behaviour and to identify promising directions for improvement of management skills. Farmers are considered intentional agents who have goals and develop and execute a coherently organized set of actions to achieve these goals. The actions are determined and executed at appropriate moments dictated by the dynamic environment. This article presents a conceptual framework that provides a means to methodically examine the processes in which knowledge and information are used by farmers when making operational decisions. Several cognitive processes are involved, including perception, interpretation, goal reasoning, planning and judgment. Farmers use them continually to address the farming, ecological, economic and social pressures they encounter. The main issue for an individual farmer is the adequacy of the instances of these processes and available information with regard to their specific performance criteria and situation. Operational decisionmaking (process-oriented) is considerably different from design or strategic decision-making (configuration- and organisation-oriented), which is "one-shot" and stated in a static way. Another characteristic of operational decision-making is that much of the knowledge invoked is tacit and internal in the farmer's mind.

\section{Cognitive Structures and Functions Involved in Agricultural Decision-Making}

Farmers' operational decision-making can be regarded as a process that ensures continual interaction with the production system and judgment about whether to postpone intervention or to act immediately, which entails considering what to do and how to do it. This decision-making process involves several higherlevel cognitive functions (called "macrocognition" by Klein et al. [7]) that convey fundamental information-processing and reasoning abilities which farmers use in natural settings. These functions manipulate or produce cognitive structures that hold farmers' beliefs, goals, plans and preferences. They include:

- attention and perception (what to attend to in the production system, the information sought, how to access the information)

- sense-making by interpreting cues (significance of the facts, identification 
and explanation of an abnormality), forming beliefs (making predictions) and recognising similar situations

- memorising and recalling important facts, events and chronologies

- formulating and selecting goals

- generating plans that correspond to these goals or adapting existing plans

- evaluating the "goodness" of a plan by mentally simulating its execution

- selecting an action based on adopted plans and current preferences

The rest of this section further describes the potential role of these cognitive functions.

Information is required throughout the production-management process, from assessing situations to evaluating decisions. To analyse decision-making behaviour it is important to consider which factors prompt farmers to seek information and then to stop their search, and which features in the production system they monitor or observe. Attention is the behavioural and cognitive process that selectively focuses on a specific aspect of information, whether subjective or objective, while ignoring other perceivable information. Perception is the identification, search and organisation of information to obtain a useful snapshot of the situation. From life-long experience, farmers develop the ability to notice specific aspects of a situation and to examine them for their potential usefulness. In other words, their experience has created certain tacit standards or norms for expectations based on previous observations. Farmers' perceptions of the production system and the surrounding environment strongly influence their decision-making process. Some of the information needed is related primarily to the farmer's goals. Information might be specified within the plan through epistemic actions (observations) or feasibility/appropriateness conditions required to execute a given action. Managing crop production is essentially overseeing trends in crop growth by frequently comparing the perceived state to the expected growth pattern. In addition to observing specific features of the biophysical system, farmers also must verify that machinery is functioning properly, ensure that necessary input resources are or will be available on time and detect abnormal conditions. Farmers must carefully select what information to search for to maintain a rapid decision process. The selection process is based on several easily available heuristics resulting from the farmer's experience, but also involves reasoning when a problem is suspected to occur.

The farmer's perception of information is useful only after it has been transformed into situational awareness, i.e., detecting patterns that help to understand the current situation (hindsight) and predicting how the situation might change (foresight). Sense-making incorporates knowledge from several domains, including animal and plant pathology, agronomy, ecology and farming systems. Most of this knowledge is tacit in the farmer's mental model. Situation assessment often entails determining whether everything is as expected or whether potential anomalies have occurred. An initial step in sense-making is identifying that decision-relevant events have occurred. These events are essential because they trigger or reactivate the decision-making process. Events may result from 
the farmer's perceptions, from significant changes within the production system detected by sensors or visual observation (e.g. fungal infestation symptoms) or from the external environment (e.g. weather or pest infestation alert). Sensemaking also involves processing information to combine several sources of data or to reframe the data into a readily available indicator, i.e., a synthetic piece of decision-relevant evidence. It may be necessary to retrieve the data from historical records that were collected from observations or from external sources (e.g., advisory services). Farmers' sense-making generates beliefs about what they detect or infer from their situations. Distinct decision-making behaviours exist because individuals have different beliefs about the same context.

An individual's ability to remember influences the ability to selectively use or ignore information in a system or the environment. The ability to encode, store, retain and subsequently recall information and past experiences differs greatly among individuals. The structures and practices used to temporarily store and manipulate information play important roles in comprehension and problemsolving. Remembering past experiences and the ability to recall previously learned facts, experiences, impressions, skills and habits improves the quality of decisions and can save time. A poor memory can influence attention because it hinders observation of correlations between facts, and useful cues are missed as a consequence. The cognitive process of recognition is essential in dynamic-situation decisions and primarily uses the ability to recall patterns from similar past situations to develop a consistent explanation of the current situation and the potential consequences. The act of memorising essentially focuses on sensed (i.e., visual, auditory, olfactory, tactile) information and the chronology of events, facts, changes, actions or procedural steps.

Farmers' professional proficiency is also embedded in their beliefs about what is desirable and reasonably expected to result from a given technical intervention. The farmer can then use this knowledge to implement a form of goal reasoning to support operational decision-making. Production-management behaviour is largely determined and explained by the crop or animal growth goals that need to be met in a production cycle. A goal is a combination of desired system features that are organised in time. Goals can be positive (attain or maintain desirable situations) or negative (prevent or mitigate undesirable situations). Motivation can be intrinsic (e.g. enjoying outdoor nature-related activities), high aspirations (e.g. ensure sufficient profit) [8] or development of a high-level target (e.g. crop yield goal) into more specific subgoals (e.g. maintaining soil productivity, maintaining necessary soil moisture throughout the growing season, ensuring labour is available at harvest). The farmer's goals can fluctuate: certain goals enter the queue while others are replaced with more explicit goals or disappear due to obsolescence caused by changes in the context. Farmers often must address a multitude of objectives, many of which conflict because progress in one comes at the expense of another. The goals may include incommensurable sources of motivation that prevent identification of an objective optimal choice. Organising the reasons in favour or against competing goals is a heuristic 
way to address potentially incomparable and conflicting situations. In practice, no formal deliberation occurs, and farmers usually just try to find an acceptable compromise. We want to understand the reasons farmers select or exclude competing goals, i.e., which goals are reasonable to adopt given the decisionmaking context, knowledge and situational information. Adopting a goal depends primarily on three factors: the relative subjective value of the goal itself, the ability to develop a plan that uses a previously learned strategy to meet the specific goal, and commitments to other plans.

A production system manager chooses or designs appropriate plans to achieve specific goals, i.e., actions with greater potential to generate a more acceptable situation. Planning is also one way to anticipate the future and guide and organise future activities. With experience, farmers attribute meaning to cumulative observations and design crop management practices as a set of purposeful technical interventions that are performed in specific situations. Management practices remain similar but are not necessarily identical each year, as situations are rarely perceived in exactly the same way. Regularity is sufficient to avoid the need to develop entirely new plans; farmers only need to adapt them. Plans usually are only sketchy because uncertainty precludes developing long and detailed plans for each moment in the future. Adaptations are often specified as variants or options attached to a default plan. Since the adopted plans are often partial, further planning is required later to adapt or refine them. Planning is based on experience and regular practices that allow for exceptions. Hence, plans are conditional and they can be reconsidered with new evidence or with new developments in the situation. Adaptable plans and reconsidering intentions are essential to manage various situations and unanticipated events.

A flexible plan does not specify completely beforehand the actions that will be executed. It merely restricts the set of admissible decisions that concern the timing of actions, the resources (labour, equipment) that can be used to execute the actions [9], and choices among alternative actions (along with the resources allocated to them) when several exist. The latter choice also involves heuristics that are applied in a "fast and frugal" manner [10] using Simon's concept of "satisficing" [11], i.e., the rapid search for an acceptable solution. Like for goals, farmers only occasionally assess and weigh reasons for or against each alternative action. The satisficing approach does not require complete examination and comparison of options or performance of complex optimal-stopping calculations. The heuristics used are extremely tacit and involve factors, such as whether the actions are too costly in material input and time, have long-term negative consequences, have other benefits (e.g. aesthetic, environmental, moral), or are simply convenient at the time of execution. These are personal preferences that, unlike goals, do not include the desire to attain or maintain a target state.

Considerable differences exist among decision makers in the emphasis placed on these cognitive functions (e.g. differences in perceptions), the underlying structures (e.g. complexity of plans), and the way they are combined in the decision process. This is highlighted in the next section by examining three relevant 
approaches from other domains that have not yet been applied in agriculture. In agriculture, these differences describe farmers' decision-making styles, i.e., their personalities as decision makers, which are described in later sections.

\section{A review of Three Fundamental Approaches to Operational Decision-Making}

Several theoretical frameworks providing effective ways to analyse management activities and managers' behaviours have been developed in domains other than agriculture, such as manufacturing, the military and emergency management. These frameworks provide the terminology and conceptual principles for understanding decision-making behaviour. In this section we briefly review three of these approaches: cognitive work analysis [12], developed by ergonomists and human factors psychologists, naturalistic decision-making [13] developed by cognitive psychology scientists, and practical reasoning approaches [14] that have roots in philosophy, cognitive science and artificial intelligence. These approaches adopt different perspectives and are based on different foundations. They also differ in the types of decision problems they can address and the factors they emphasise.

These approaches focus on identifying and describing what shapes the decision-making process that governs the choice of actions in a professional domain. The agent usually must decide between alternative actions in light of the goals, consider potential consequences of and conflicts among the goals, and decide whether to act or do nothing. Each approach developed specific conceptual descriptions of the components and possible functioning of the process in which agents make decisions and, ultimately, act in the context of their natural environment.Finally, complete content and organizational editing before formatting.

\subsection{Decision Ladder}

Rasmussen (1976) created the decision ladder (DL) method to represent cognition in the domain of process control. A DL is a graph of nodes and links that breaks down decisions and subsequent follow-ups in response to changes in the decision maker's states of knowledge (beliefs) about a problem-solving task. The DL helps to identify the requirements for decision making [15] and provides a useful way to break decision making down into a set of generic activities that underlie the fundamental sequence of stages from situation analysis to target formulation, and, ultimately, to planning and execution of actions [16]. In other words, the decision maker examines the process involved in identifying whether a situation requires action, analyses the situation and evaluates its consequences, determines a target state, devises a course of action to achieve this target state, and executes the procedure developed. The DL includes behaviours that can occur under different conditions, for example, when experts encounter unfamiliar situations or when novices perform certain tasks. Depending on their cognitive skills and familiarity with the system, decision makers may omit certain activities of the DL. These shortcuts involve heuristics that make the process more ef- 
ficient. They are essential features to distinguish managers according to their decision-making skills. By emphasising the identification of potential cognitive states and processes involved in a given task, the DL method [17] is useful for i) designing the organisation of cognitive work in complex large-scale systems that are likely to involve several agents and ii) studying reliability and safety issues [15] [18].

\subsection{Recognition-Primed Decision Making}

Naturalistic decision making (NDM) [19] [13] focuses on how people make decisions in complex socio-technical contexts. The recognition-primed decision (RPD) model is the main protocol derived from the NDM framework. This model describes the strategy experts use to make decisions in familiar situations and under stressful conditions such as those with high risk, intense time pressure or rapid changes. In this approach, decisions are based on recognising similarities between the current situation and a previously experienced situation. Recognition encourages pursuing a course of action similar to the one that was effective on the previous occasion. The art of decision making lies here in the ability to categorise or classify situations. The RPD model focuses on how people use their experience in the form of a "repertoire of patterns" defined by relevant cues, expectations for changes in the situation, seemingly reasonable goals, and the most typical goal-related courses of actions to execute. It also represents a way to bypass certain deliberation steps as with the DL approach [17]. The RPD model involves a mental simulation activity that assesses the appropriateness of a set of chosen actions. The RPD approach aims at decision problems in which situation awareness and subtle aspects of expertise play a key role. It is associated with powerful means of eliciting expert knowledge. NDM researchers stress the importance of considering the psychological processes that generate decisions rather than focusing primarily on final outcomes. The RPD model highlights the role of mental simulation but does not describe its properties or functioning in detail.

\subsection{Belief-Desire-Intention-Based Practical Reasoning}

Reasoning about what to do is known as "practical reasoning" in philosophical studies [20] of human rationality. Practical reasoning is the inferential ability by which agents determine purposeful intentions to act and ultimately actions that agree with their subjective values and the current circumstances. The inferences depend on agents' knowledge and understanding of the particular circumstances of a specific situation. The belief-desire-intention (BDI) model [14] is possibly the best known and most studied model of practical reasoning. It was developed for software agents [21], but the theoretical framework applies equally well to human agents. This model contends that the daily activities of deliberation (deciding what to do) and means-ends reasoning (deciding how to do it) are based on the following principles. Based on an agent's beliefs (which are constantly updated with sensory input from the environment), an agent generates desires 
(goals), which are states of the world that may be worth achieving. These desires are then filtered according to their desirability and achievability to determine concrete goals to be adopted. As a result of this deliberation phase, the agent examines plans and commits to the one with the most potential, which becomes the intentions. The BDI model provides an iterative mechanism that dynamically generates and selects goals, then designs appropriate plans and ultimately chooses actions guided by these plans. Beliefs are the informative component of the decision process, while desires are its motivational component. Intentions play an essential role in restricting the set of options about which the agent must think. They involve a commitment to action and allow the agent to ignore options that do not correspond with the previously adopted intentions. Thus, the concept of intention is one way to limit the time spent deliberating about what to do. Research in this area has resulted in the axiomatisation of certain BDI models, formal logical descriptions (Wooldridge 2009) and several computer implementations and applications [22] [23].

\section{Comparison of the Three Approaches}

The three approaches differ from the rationalistic decision-making paradigm, which is an analytical approach to decision making that assumes alternative solutions can be generated and that the decision maker can judge the success of each outcome using clear and discernible criteria to find the "best" option. Each of the three approaches contributes to understanding and modelling operational decision-making in a more accurate way that grasps the structure and functioning of purposeful behaviour in a complex agricultural production context. Here, we consider their most essential features to generate a new template of operational decision-making (next section) that exploits these features and is potentially more useful than any of the three approaches considered in isolation.

The RPD and DL approaches emphasise that operational decision-making is situation-specific and defined by the type and quantity of information available and the pressure to make a decision. They postulate that non-novice decision makers use their rich experiences to identify typical situations and use analogies to implement solutions. This behaviour decreases the complexity of the problem, takes into account limited cognitive resources, and increases the efficiency of decision making. The shift in emphasis in the RPD approach originated from the "bounded rationality" concept [24], which advocates replacing a rational idealised perspective with a decision process involving mental assessment of situations, mental simulation of outcomes of possible actions and resolution of the decision by committing to actions that provide satisfactory results. While the rational perspective has been shown to describe decision making adequately in certain contexts, applying it to operational decision-making in agriculture is problematic and even impractical [25]. Rather than linearly evaluating all possible options for an optimal solution, the decision maker uses cues in the environment to obtain a set of goals and an appropriate pre-formulated procedure 
based on analogical reasoning with similar situations. The procedure is a template that must be adapted to fit specific situations. Once the procedure has been instantiated, it can be evaluated, at least qualitatively, by mental simulation.

Although the DL and RPD approaches typically yield courses of action, they do not provide specific ways to address dynamic decision problems, in which successive decisions are not independent and require an iterative "look-ahead" approach. In other words, these approaches primarily address short-term goals. In contrast, the BDI approach focuses on dynamic decisions in which goals and beliefs change continuously. For this reason, the BDI approach separates and articulates deciding which beliefs and goals to establish (deliberation) and how to achieve the goals (means-ends reasoning). Simon [11] argued that each type of serious, complex decision involves deliberate (i.e., causality-based) and actionoriented thought. Actions can sometimes be decided in isolation, but they usually need to be considered as a set of actions organised within a planning process. This is necessary when certain actions depend on previous ones, as occurs in agricultural production, and when resources (machinery or labour) may be limited at execution time. The simplest plans are sequences of actions arranged in chronological order, but plans may also be logically complex [26], including disjunctions or conditions that restrict adapting or executing part of the plan to specific situations. In particular, plans can involve conditional forks because it is not known exactly which situation will occur when it is time to execute a given stage. Each action in a plan may be subject to conditions that depend on the current situation and the outcome of previous steps.

The concept of intention is the most distinctive feature in the BDI approach. Here, we describe intentions in more detail and explain their relevance to studying operational decision-making in agriculture. In general, the concept of intention is a mental state that represents commitment to a plan that is expected to fulfill a goal. It guides an agent in attempting to meet a specific goal. According to Bratman's "theory of agency" [20], the primary function of an intention is pragmatic, because the forming of prior intentions encourages the agent to connect the chosen goal to partially specified actions coordinated over time, and these combined actions are the result of deliberation. Intentions drive meansends reasoning: when one adopts an intention, one will try to achieve it, which influences the choice of action. Intentions create a more predictable future by committing the agent to act consistently with plans that partially anticipate the future. In the long run, the planning support that accompanies intentions generates more consistent (less erratic) management decisions than approaches based solely on beliefs and desires. Intentions persist: once adopted, they are maintained until goals are met or deemed unachievable. Once an intention is formed, the agent usually will not continue to deliberate; options that are inconsistent with intentions will be discarded. However, intentions may be abandoned or modified depending on external events or changes in the situation. In other words, the intention governs the operational decision-making behaviour unless an event requires revising the current intention Intentions influence observa- 
tions of the agent, who must at least monitor properties of the conditions used in the adopted plan. In the period between forming an intention and taking action, new goals and new intentions may be generated; existing intentions may be further specified in partial plans, which must be completed as new information becomes available. In particular, to generate action from an intention, the agent must decide on the intended means; therefore, intentions also prompt decisions about means. Thus, intentions provide a background that allows the agent to adapt the temporal horizon of deliberation while simultaneously narrowing its scope to a smaller set of possibilities.

Pollock [27] [28] incorporates Bratman's intentions, but adds a new component to practical reasoning, which he calls "likings" or values of the decision maker. Pollock assumes that the function of rationality is to create a more acceptable world for the individual. A rational agent must have a way to discern how likable a situation is, which is a feeling produced by the agent's beliefs about the situation [29]. Humans are introspectively aware of these feelings, which play an essential role in deliberating about goals, plans and actions.

\section{Conceptual Modelling}

The conceptual framework connects the decision system (the farmer) to the farm agro-ecosystem via actions (Figure 1). Both systems exist in a climate and socio-economic environment that includes other farms, advisory services, and agro-business organisations in a given region. The farmer is considered a system that observes (epistemic actions) and transforms (pragmatic actions) the agroecosystem using the available resources. The farmer system is composed of seven interactive components: six are repositories for beliefs, desires (goals), intentions, plan components, preferences and events manipulated by the farmer. The remaining element is a system that embodies the farmer's mental model of the agro-ecosystem's components and their relationships, its internal functioning

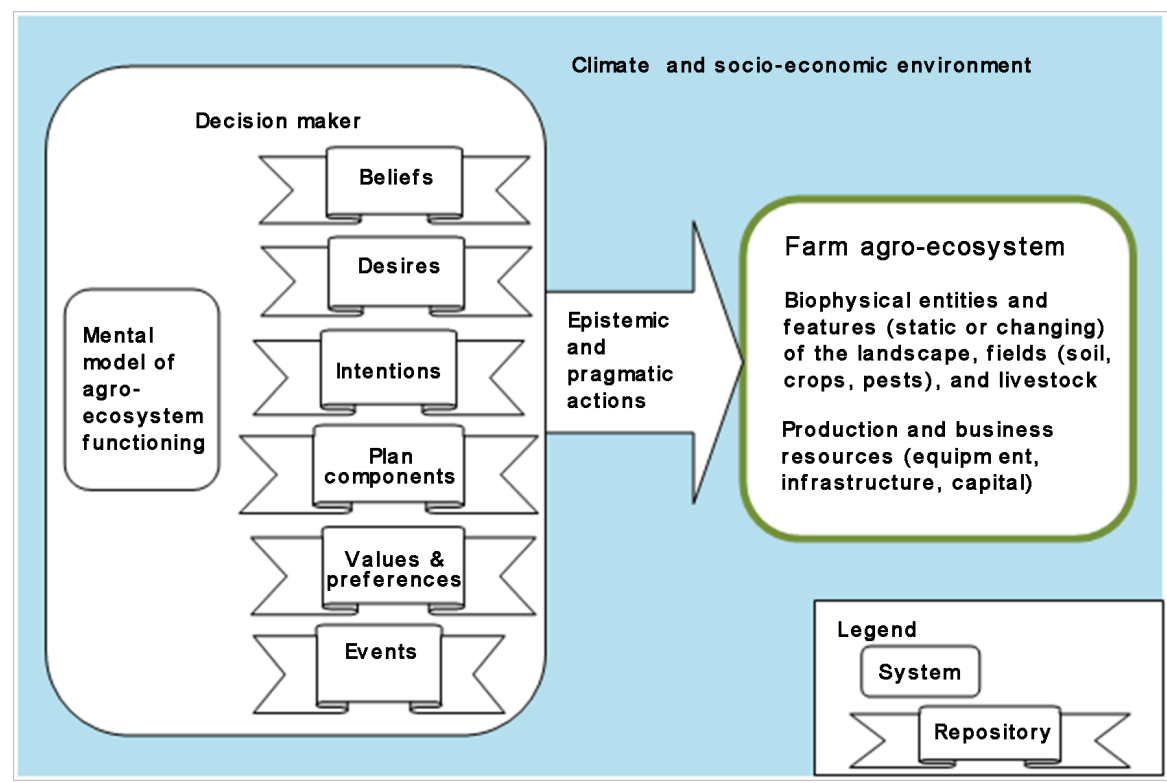

Figure 1. The farm production system within the surrounding environment. 
and its expected responses to management actions. The mental model also includes a range of knowledge or beliefs about concepts (disease or pest infestation), images of these concepts and causal relationships between events.

Beliefs, goals, and intentions change constantly as new observations and events occur; previous goals may be abandoned while new goals are added, and intentions are likely to be adapted or revised. Values and preferences play a key role in the decision-making process because farmers frequently must choose among alternative goals, plans and actions. Values and preferences also can change, although they tend to be more stable than beliefs, goals and intentions. They may, however, be sensitive to the farmer's emotional or physiological state (e.g. stress, fatigue). Plan components are the building blocks used to develop plans; each component has conditions that define the situation patterns in which the component is potentially relevant. Decision making in a dynamic environment requires adaptation to address incidental or expected events that the farmer considers significant. Events come from external sources (e.g. weather event, pest infestation) or are generated as a fulfillment (logical consequence) of certain processes, such as the end of work. They can also be identified as distinct stages of a process, such as a crop's change in growth stage. Certain events are known in advance and can be marked on a calendar. Of importance in operational decision-making are the incidental events that occur when tasks are interrupted, by bad weather for example, which requires contextual adaptation. Events play an essential role in dynamics of the decision process because they trigger or reactivate cognitive functions.

Reflective decision-making behaviour iterates a chain of cognitive processes (Figure 2), which interact with the information repositories (Figure 1), from

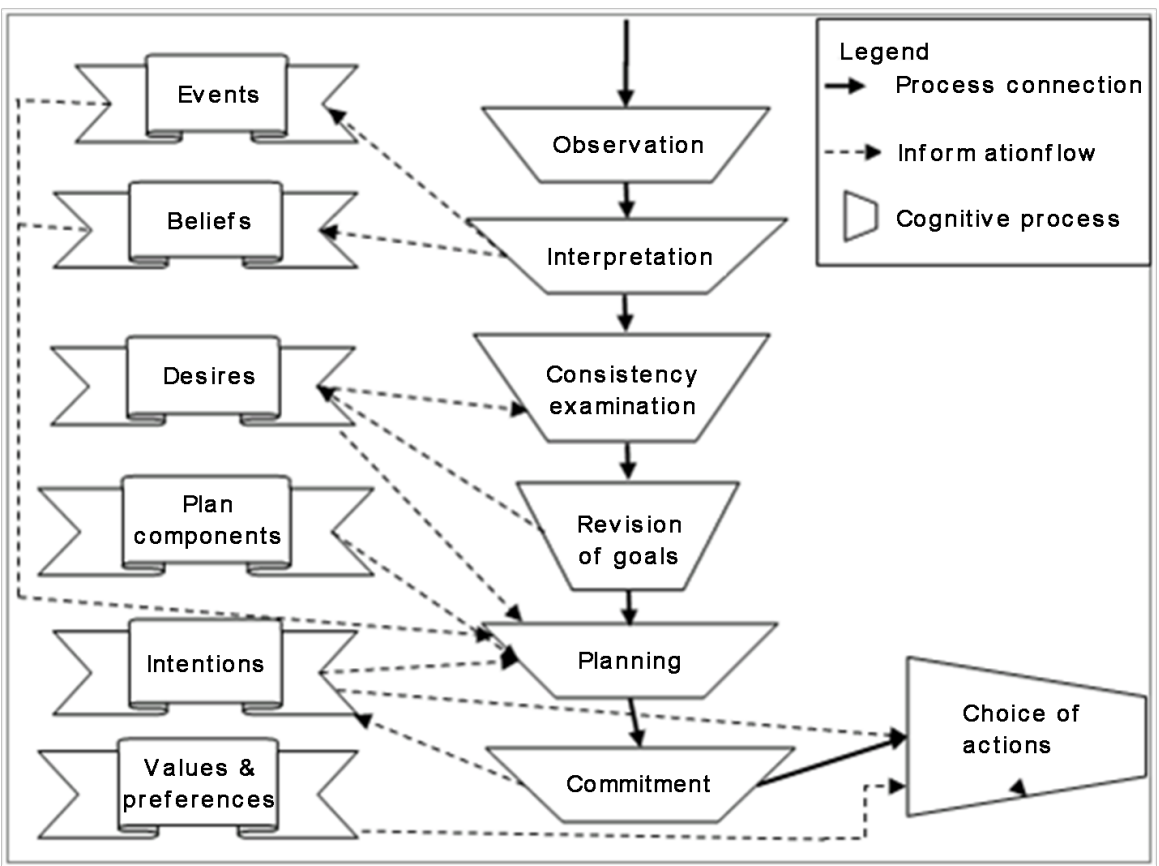

Figure 2. Basic chaining of the cognitive processes in reflective behaviour. Processes underlying the mental model shown in Figure 1 are omitted. 
observations to decisions about technical interventions. The decisions induce changes that also result from the biophysical system's own internally generated dynamics. Basically, a farmer forms beliefs based on perceptions of the situation, envisions desirable future situations, develops plans to create a more acceptable world, and performs actions to follow the intended plans. More specifically, farmers' cognitive processes involve the following continuous cycle:

1) Observation, which is the collection of information about specific features of the agro-ecosystem and its environment or specific events perceived as important for management. The farmer monitors the agro-ecosystem and its environment for management-relevant cues. Perception activities are the part of the management plan that contains epistemic actions, most of which are routinely performed.

2) Interpretation, which involves analysing the situation to recognise abnormal situations, assessing the situation (finding causes that can explain it), and predicting how the situation will change given no intervention. Farmers must be aware of information that is potentially significant and worthy of interpretation. The outcome of this step depends greatly on the farmer's mental model of internal dynamics of the agro-ecosystem. Memorised information about past events and dynamics of certain key biophysical variables play an important role here.

3) Examination of the compatibility and appropriateness of current goals given the current situation and the previous history. This consistency examination phase also uses the mental model of the agro-ecosystem.

4) Modification of existing objectives, which might result in abandoning operations, when objectives are no longer relevant or feasible; adding operations, when deliberation reveals desirable and feasible features; or adapting operations, to replace general goals with more specific goals.

5) Planning: changes in goals and situations will naturally induce updating or revision of intentions. This requires examining existing plans in the repository of plan components and tentatively adapting or developing new ones, if needed. This exploration involves a kind of mental simulation based on the farmer's mental model.

6) Commitment: Ultimately, the farmer commits to certain outcomes of the planning phase.

7) Choosing actions, which involves resolving conflicts among competing goals, concurrent intentions and work resources in short supply [9] and personal preferences are invoked to decide.

In operational decision-making, the course that farmers follow is continuously generated and regenerated from inside the system.

Decision making (Figure 2) is reactive to events and proactive in the sense that it includes anticipatory deliberation of what might happen and what actions can be planned to shape the future in the desired direction, organise work in advance, be ready to exploit opportunities and take preemptory actions against potential problems and threats. The conceptual framework (Figure 2) provides a 
general map of the components, processes, and input and output. These ingredients can then be used to construct a model of a decision maker. The framework is a template that can be particularized differently according to different decision behaviours. Cognitive functions in natural decision-making settings differ significantly among farmers. For example, planning may span a longer or shorter horizon in the future. The plan component repository may consist of a set of simple rule-based associations between situation patterns and appropriate actions or more detailed procedure-like constructs that involve temporal and conditional characteristics [26] [30]. The process by which values and preferences are combined to produce the chosen actions can also vary among farmers, although little is known about this process. In addition, the way they are combined and the importance of the role of each function are also distinguishing features in decision making. For example, not all farmers distinguish goals of primary interest from goals with instrumental utility (i.e., required to achieve other goals), or reason about the consistency of a set of goals in a given situation. Certain cognitive functions simply seem to be absent or nearly invisible in certain cases (Figure 3), representing behaviour based on rigid pre-set intentions and nearly no adaptation. In the example of Figure 3, observation and interpretation are simply used to recognise crop stages that are referred to in the plan adopted and intended at the initial (design) stage of the crop production process. Decision making is rather straightforward in this case.

\section{Discussion and Conclusion}

The conceptual framework in the previous section is a template for developing a process-oriented view of decision making. It relies primarily on the rational

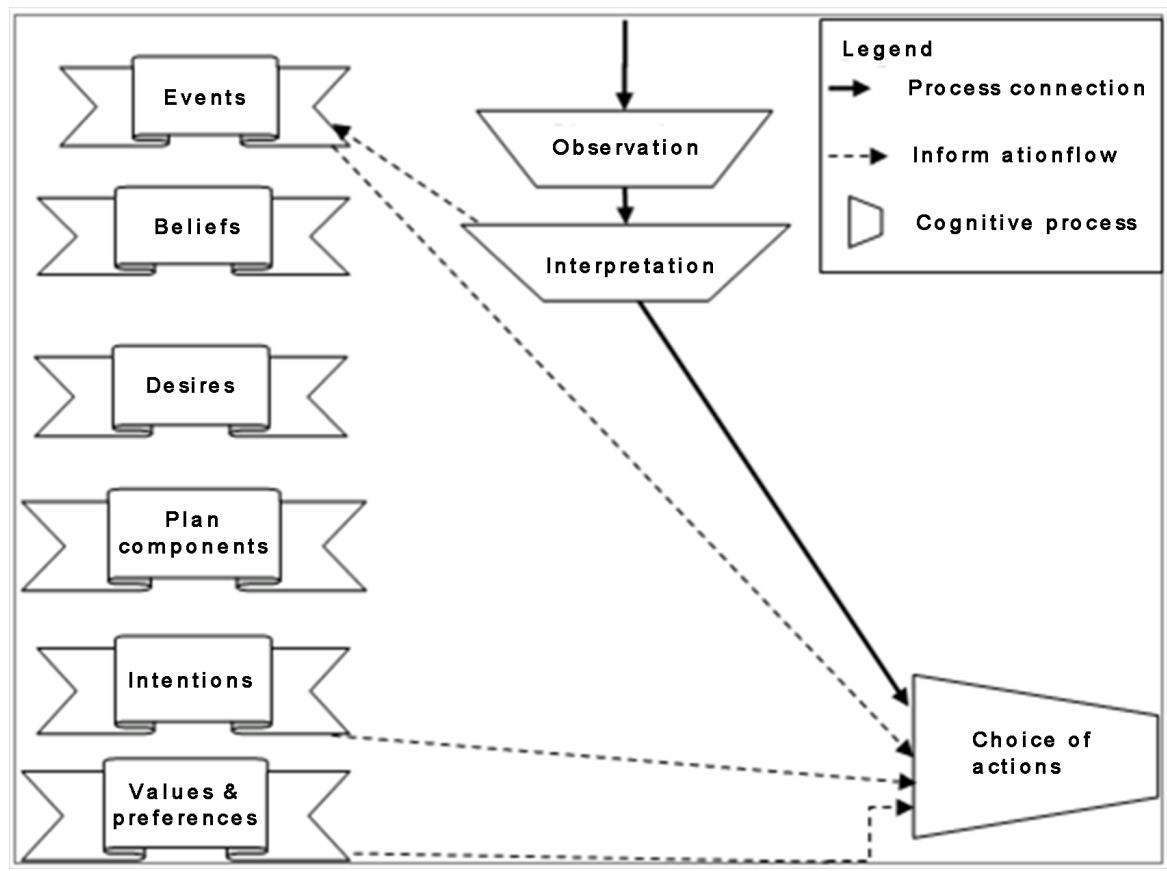

Figure 3. Non-adaptive behaviour based on rigid pre-set intentions. 
agency promoted by BDI-based architecture and incorporates key ideas from the DL and RPD models that focus on the role of heuristics in operational decision-making. Perceptions and desires, combined with a mental model of system functioning, lead iteratively to actions via a chain of cognitive processes. Understanding the process by which individuals make decisions is essential for understanding their decisions.

We believe that farmers' behaviours cannot be explained without understanding their mental processes. Analysis must begin with analysing the functions and activities that occur or may occur to achieve goals given their constraints and personal features. Our current understanding of how individuals process information remains in a formative stage. Focusing on the content and organisation of the cognitive functions an individual uses in the decision process can generate a model-based investigation of farmers' operational decisionmaking. The presented framework provides a way to describe farmers' decision styles (i.e., patterns of information processing). It is nevertheless only the beginning of an adequate foundation for research on farmers' decision behaviour in the technical management of an agricultural production process.

Conceptual models of decision making (Figure 2 and Figure 3) emphasise the roles of cognitive functions. The potential influence of the mental model of agro-ecosystem functioning (Figure 1) is also extremely important. Farmers who experience difficulty anticipating side effects of their interventions and integrating them into their thinking processes may have inadequate mental representations of the systems they are trying to manage. Mental models result from the tendency to form ad-hoc explanations of events and functioning in the biophysical world. The features that induce complexity include feedback mechanisms, non-linear relationships between variables, random disturbances, irreversible processes, adaptive processes, and time lags. It is critical for farmers to understand these complexities and consider them in the planning process to have adequate performances and decreased production risk, especially during transitions in the farm business (e.g. new crops or new technical practices).

Until we understand the causes of poor decision-making and performance in complex, dynamic decision environments, it will be difficult to design interventions and strategies to correct shortcomings. Simulation can provide a virtual arena for experimental investigation despite the ineffability of a large part of the knowledge which farmers use in operational decision-making. Simulation can help to explore consequences of several sources of failure including:

- inaccurate mental models or over-simplification

- relevant information not being collected (consciously or unconsciously)

- poor interpretation of information or discounting of conflicting information

- failure to revise goals in light of new evidence

- failure to revise intentions in light of new evidence

Many modelling attempts have been made, but they address only partially the type of cognitive considerations discussed in this article. Most address patternbased decision-making approaches that implement reductionist behavioural 
theories based on reactive rules. Few have considered realistic representations of plans, their event-dependent adaptation or adequate operational decision-making processes that respond to actual situations and resource availability. Models rarely consider goal reasoning or elicitation, representation and processing of preferences.

The conceptual model in this article has several limitations. One limitation is the omission of mechanisms that induce farmers to change their perceptions and behaviour, i.e., the mechanisms of learning. Learning is the combination of processes individuals use to improve their decision behaviour. Learning could provide changes in ( $\mathrm{i}$ the way individuals think about system functions (the mental model), (ii the observed cues and ways they are interpreted, (iii the goals they are trying to meet and (iv the plan components they use. Another limitation is the overly restrictive view of the farm decision-maker as a single agent. Farm management tasks are often performed by several members of the household and therefore involve a social dimension that is difficult to define. Social aspects of decision making also need to be addressed when several farmers share material resources (equipment or irrigation water) or have common goals (create or maintain ecosystem services at the landscape scale). This requires coordination of their respective plans and actions. More research is needed to create a solid foundation to integrate the key concepts, processes and issues identified in this article.

In its present form, the conceptual framework provides us with an articulate decomposition of decision behavior components and mechanisms. Once particularized for a specific case, it facilitates analysis and structured discussion between farmers, extension agents and researchers. The next step aims at embedding the conceptual framework into an agent-based computational framework that can support the implementation of agroecosystem models (including their human components) and, then, deeper investigation through virtual experimentation.

\section{References}

[1] Hardaker, J.B., Huirne, R.B.M. and Anderson, J.R. (1997) Coping with Risk in Agriculture. CAB International, Wallingford.

[2] McCown, R.L. (2005) New Thinking About Farmer Decision Makers. In: Hatfield, J.L., Ed., The Farmer's Decision: Balancing Economic Successful Agriculture Production with Environmental Quality. Soil and Water Conservation Society. Ankeny, Iowa, 11-44.

[3] Edwards-Jones, G. (2006) Modelling Farmer Decision-Making: Concepts, Progress and Challenges. Animal Science, 82, 783-790. https://doi.org/10.1017/ASC2006112

[4] Feola, G. and Binder, C. (2010) Towards an Improved Understanding of farmers' Behaviour: The Integrative Agent-Centered (IAC) Framework. Ecological Economics, 69, 2323-2333. https://doi.org/10.1016/j.ecolecon.2010.07.023

[5] An, L. (2012) Modelling Human Decisions in Coupled Human and Natural Systems: Review of Agent-Based Models. Ecological Modelling, 229, 25-36. https://doi.org/10.1016/j.ecolmodel.2011.07.010 
[6] Schlüter, M., Baeza, A., Dressler, G., Frank, K., Groeneveld, J., Jager, W., Janssen, M., McAllister, R., Müller, B., Orach, K., Schwarz, N. and Wijermans, N. (2017) A Framework for Mapping and Comparing Behavioural Theories in Models of Social-Ecological Systems. Ecological Economics, 131, 21-35. https://doi.org/10.1016/j.ecolecon.2016.08.008

[7] Klein, G.A., Ross, K.G., Moon, B.M., Klein, D.E., Hoffman, R.R. and Hollnagel, E. (2003) Macrocognition. IEEE Intelligent Systems, 18, 81-85. https://doi.org/10.1109/MIS.2003.1200735

[8] Öhlmér, B., Olson, K. and Brehmer, B. (1998) Understanding Farmers' Decision Making Processes and Improving Managerial Assistance. Agricultural Economics, 18, 273-290. https://doi.org/10.1016/S0169-5150(97)00052-2

[9] Martin-Clouaire, R. and Rellier, J.-P. (2011) Dynamic Resource Allocation in Farm Management Simulation. In: Chan, F., Marinova, D. and Anderssen, R.S., Eds., Proceedings of MODSIM2011, Perth, 12-16 December 2011, 1652-1658. www.mssanz.org.au/modsim2011/B1/martin clouaire.pdf

[10] Gigerenzer, G. (2004) Fast and Frugal Heuristics: The Tools of Bounded Rationality. In: Koehler, D. and Harvey, N., Eds., Blackwell Handbook of Judgment and Decision Making, Blackwell, Oxford, 62-88. https://doi.org/10.1002/9780470752937.ch4

[11] Simon, H.A. (1996) The Sciences of the Artificial. The MIT Press, Cambridge.

[12] Rasmussen, J. (1976) Outlines of a Hybrid Model of the Process Plant Operator. In: Sheridan, T.B. and Johannsen, G., Eds., Monitoring Behaviour and Supervisory Control, Plenum, New York, 371-383.

https://doi.org/10.1007/978-1-4684-2523-9 31

[13] Klein, G.A. (1998) Sources of Power: How People Make Decisions. MIT Press, Cambridge.

[14] Bratman, M., Israel, D. and Pollack, M. (1988) Plans and Resource-Bounded Practical Reasoning. Computational Intelligence, 4, 349-355. https://doi.org/10.1111/j.1467-8640.1988.tb00284.x

[15] Vicente, K.H. (1999) Cognitive Work Analysis: Towards Safe, Productive, and Healthy Computer-Based Work. Lawrence Erlbaum, Mahwah.

[16] Rasmussen, J., Petjersen, A.M. and Goodstein, L.P. (1994) Cognitive Systems Engineering. Wiley, New York.

[17] Lintern, G. (2010) A Comparison of the Decision Ladder and the Recognition-Primed Decision Model. Journal of Cognitive Engineering and Decision Making, 4, 304-327. https://doi.org/10.1177/155534341000400404

[18] Hassall, M.E. and Sanderson, P.M. (2014) Can the Decision Ladder Framework Help Inform Industry Risk Assessment Processes? Ergonomics Australia, 10, 3.

[19] Klein, G.A. (1989) Recognition-Primed Decision. In: Rouse, W., Ed., Advances in Man-Machine Systems Research, JAI Press, Hartford, 47-92.

[20] Bratman, M. (1987) Intentions, Plans and Practical Reason. Harvard University Press, Cambridge.

[21] Wooldridge, M. (2009) An Introduction to MultiAgent Systems-Second Edition. John Wiley \& Sons, Hoboken.

[22] Son, Y.-J. (2014) An Extended BDI-Based Model for Human Decision-Making and Social Behavior: Various Applications. In: Ceberio, M. and Kreinovich, V., Eds, Constraint Programming and Decision Making, Volume 539 of the series Studies in Computational Intelligence, Springer, Berlin, 171-174. https://doi.org/10.1007/978-3-319-04280-0 19 
[23] Blake, T. and Gilbert, N. (2014) How Do Agents Make Decisions? A Survey. Journal of Artificial Societies and Social Simulation, 17, 13. https://doi.org/10.18564/jasss.2687

[24] Simon, H.A. (1955) A Behavioral Model of Rational Choice. The Quarterly Journal of Economics, 69, 99-188. https://doi.org/10.2307/1884852

[25] Daydé, C., Couture, S., Garcia, F. and Martin-Clouaire, R. (2014) Investigating Operational Decision-Making in Agriculture. Proceedings of the 7 th International Congress on Environmental Modelling and Software, San Diego, 15-19 June 2014.

[26] Martin-Clouaire, R. and Rellier, J.-P. (2009) Modelling and Simulating Work Practices in Agriculture. International Journal of Metadata, Semantics and Ontologies, 4, 42-53. https://doi.org/10.1504/IJMSO.2009.026253

[27] Pollock, J.L. (1995) Cognitive carpentry. Bradford/MIT Press, Cambridge.

[28] Pollock, J.L. (2000) Rational Cognition in OSCAR. In: Jennings, N. and Lesperance, Y., Eds., Proceedings of ATAL-99 Lecture Notes in Computer Science, Springer Verlag, Berlin. https://doi.org/10.1007/10719619 6

[29] Pollock, J.L. (2006) Thinking about Acting: Logical Foundations for Rational Decision Making, University Press, Oxford. https://doi.org/10.1093/acprof:oso/9780195304817.001.0001

[30] Martin-Clouaire, R., Rellier, J.-P., Paré, N., Voltz, M. and Biarnès, A. (2016) Modelling Management Practices in Viticulture while Considering Resource Limitations: The Dhivine Model. PLoS ONE, 11, Article ID: e0151952. https://doi.org/10.1371/journal.pone.0151952

\section{Submit or recommend next manuscript to SCIRP and we will provide best service for you:}

Accepting pre-submission inquiries through Email, Facebook, LinkedIn, Twitter, etc. A wide selection of journals (inclusive of 9 subjects, more than 200 journals)

Providing 24-hour high-quality service

User-friendly online submission system

Fair and swift peer-review system

Efficient typesetting and proofreading procedure

Display of the result of downloads and visits, as well as the number of cited articles

Maximum dissemination of your research work

Submit your manuscript at: http://papersubmission.scirp.org/

Or contact as@scirp.org 